\title{
Ageism: Lack of Implicit Stereotypes Across Adulthood
}

J essica Linberts and Andrea J une, co-authors

pp. 83-94.

Oshkosh Scholar, Volume I, April 2006

Copyright $@ 2006$ University of Wisconsin Board of Regents

All rights reserved. No part of this journal may be reproduced in any form without the permission of University of Wisconsin Oshkosh

University of Wisconsin Oshkosh

Office of Grants and Faculty Development

800 Algoma Blvd.

Oshkosh, WI 54901

(920) 424-3215

www. uwosh.edu/grants 


\title{
Ageism: Lack of Implicit Stereotypes Across Adulthood
}

\author{
Jessica Linberts and Andrea June, co-authors \\ Kathleen Stetter, Psychology, faculty adviser
}

\section{Abstract:}

This study expanded the sample populations which have been tested for ageism. An encoding task and an unexpected recall task measured ageism within these populations: 18-25 years, 25-54 years, and 55+ years. Eighty-seven participants were tested. There was no significant difference between mean percent of words, positive or negative, recalled from the old encoding task and words, positive or negative recalled from the young encoding task in the separate age categories. This supports research showing ageism does not exist in the current population. Overall, mean percent of positive words recalled was significantly higher than negative words recalled. Mean percent of positive words recalled in the self referent encoding task was significantly higher than negative self referent. Further research exploring alternative methodologies for testing implicit ageism in these populations is recommended.

\section{Introduction}

Stereotypes help to guide us through a very complex and detailed world by generalizing information. However, sometimes these generalizations can take on a negative connotation. These are then referred to as prejudices. Two highly researched types of prejudices are sexism and racism. The PsycInfo Database lists more than 32,340 journal articles related to either sexism or racism that have been published from 1900 to 2005. More recently, some research has focused on a different type of -ism: ageism. "Ageism, like sexism and racism, is the prejudice behavior of a society against older adults or negative stereotyping" (Hoyer \& Roodin, 2003, p. 39) of the aged. Negative stereotyping against the aged has been depicted as the "new American bigotry" by Butler (1978) who first defined the term ageism as stereotyping and discriminating against people based on age (Cited in Sorgman \& Sorensen, 1984, p. 18). Ageism, when imbedded in a society, allows younger generations to view older adults differently from themselves; as a result, they cease to relate to them as human beings (Butler, 1978, p. 12).

Research on ageism is important because the life expectancy of the average person in the United States is increasing due to advances in both medical and industrial technology. According to current population trends, by the year 2030, older adults will make up approximately $20 \%$ of the population (Erber, 2005, p. 16). Compared to the population pyramids for the United States in the years 1900, 1970, and 1995, which display a traditional pyramid shape with 0 to 5 year olds at the base, 2030 is projected to show a more even distribution across all age intervals (Erber, 2005, p. 18).

Disturbingly, a study examining the instances of the phrase "senior moment" (Bonnesen \& Burgess, 2004, p. 123) in newspapers between the years of 1991 and 2000 revealed the term was used 181 times in 136 newspaper articles and became more common in the latter years. The phrase "senior moment" (p. 123), although most commonly defined as brief slips in memory, can also be indicative of serious cognitive and functional deficiencies. The fact that it is referred to as a "senior" (p. 123) moment 
combined with the increasing popularity of the phrase suggests that negative stereotypes of older adults are becoming more accepted in American culture. As stated by Hawkins (2004), “....as the elderly population continues to grow, it is important that we learn more about attitudes toward the elderly. By gaining a more thorough understanding of these attitudes, we can plan and evaluate interventions aimed at improving them" (p. 278).

The research on ageism in the last few decades has reported conflicting results. Tuckman and Lorge conducted one of the first systematic studies of attitudes toward older adults. The study asked graduate students questions targeting older adults (Cited in Slotterback, 1996, p. 552). Results from this study show that these graduate students held negative stereotypes toward the aged. Since then, several methodologies and scales have been used to study ageism. The Aging Semantic Differential Scale has received wide use in assessing the attitudes and perceptions of college students toward older adults. This scale pairs bipolar personality traits requiring the participant to indicate the trait more representative of an older adult. The results of the studies using this scale are inconsistent. For example, Bell and Stanfield's (1973) study indicated non-significant results of positive or negative attitudes toward aging while Hawkins (1996) found that students hold negative views about the aged. Aaronson (1966) administered the Grough Adjective Rating Scale (GARS) to participants where he asked them to "check those adjectives which, in their opinion, described the typical person at decade intervals from 5 through 85 " (p. 458). Then he tabulated the frequency with which more negative words were checked in relation to the older ages. With the increase in age, more negative words were checked which indicated age biased attitudes. Weinberger and Millham (1975) assessed attitudes of undergraduate students using an attitude questionnaire in regards to two age categories: a 25-year-old category and 70-year-old category. The participants then rated the target age for various attitudes on an ordinal scale called the Likert scale (p.343). Their results indicated participants held ageism toward the group of older adults but gave more positive responses regarding a personalized older person, such as a grandmother. Mosher-Ashley and Ball (1999) had participants complete a three-page survey modified from one developed by Sheomake and Rowland (1993), which included a checklist format of a series of characteristics. Mosher-Ashley and Ball found undergraduate students tend to hold more positive views toward the aged. Other studies, like one done by Middlecamp and Gross (2002), focus on how intergenerational contact influences attitudes about older adults or aging. The study measured children's attitudes using CATE, Children's Attitudes Towards the Elderly (p. 277). The study found despite contact with older adults in an intergenerational daycare program, preschool children rated the older adults less capable than younger adults and children of participating in the same activities.

Unlike the previously listed studies, Perdue and Gurtman (1990) used an encoding task followed by an unexpected recall to analyze if ageism might have an "unintentional and perhaps unconscious component" (p. 201). The study attempted to depict if negative traits were automatically perceived in reference to old persons when the initial information was encoded (p. 201). This and other studies of stereotyping have recently advanced from the assumption that people organize information about the world "schematically" (Carver \& de la Garza, 1984, p. 69). This means information, instead of being encoded in separate, individual pieces, is structured so that associa- 
tion between elements is represented along with the elements themselves. Associated information consistent with a person's schematic categorization at the time of encoding will be easier to store and retrieve (Perdue \& Gurtman, 1990). This also seems to be valid in reference to social groups or categories (p. 202). "One important reflection of schematic representation is the following: when one aspect of schema is activated or evoked, other related aspects of the schema tend to be evoked as well" (Carver \& de la Garza, 1984, p. 69). Therefore, when an encoding task uses the word "old," all information associated with that word, including any negative stereotypes, will be activated in the schema. "Spreading Activation" (Payne \& Wenger, 1998, p.252) is the term psychologists have given to this processing in the schema. Perdue and Gurtman (1990) utilized schema activation to identify negative implicit stereotypes about older adults and measured with an unexpected recall test.

Sample populations used for studying ageism have been limited in many studies; oftentimes, experimenters have relied upon a convenience sample of undergraduate students. However, there have been a few exceptions. For example, Middlecamp and Gross (2002) measured children's attitudes resulting from intergenerational contact, Mosher-Ashley and Ball (1999) measured attitudinal differences between traditional students and non-traditional students defined as 23 or more years, and Bell and Stanfield (1973) measured a sample of older retired adults in studying attitudes toward age. A literature review found no studies have looked at ageism across adulthood. A computerized literature search was carried out on the following databases: PsycINFO (Psychological Abstracts), (1887 to present); EBSCO host, (1985 to present); and Wilson Web, (1983 to present). Keywords used in the computer search were "attitudes," "ageism," "ageism" <in> "various populations," "ageism" <in> "adulthood," "ageism" <in> "elderly" and "ageism" <in> "aged". No hits were found. Research needs to focus on all aspects of the population who could hold negative stereotypes to fully study, understand, and address ageism in this society.

The following research is an extension of the study done by Perdue and Gurtman (1990) titled "Evidence for the Automaticity of Ageism." The purpose of this study is to determine if other age populations, aside from undergraduate college students, hold negative attitudes toward the aged-specifically non-traditional students and retired senior citizens. Our research question is: "Are discriminatory attitudes held mainly by young adults, or do middle-aged and even older adults themselves have ageist attitudes?" (Erber, 2005, p. 15). By seeking out all age populations who may hold negative attitudes toward the aged, psychologists and society can better understand and eradicate them.

We predict that in the self-referent encoding task, participants across the three age categories will recall more traits, both positive and negative, because self-referent information is processed at a deeper level as found in Rogers et al. (1977) (Cited in Rudolph, 1993). As Rogers et al. also found in the study, fewer traits, both positive and negative, will be recalled in the evaluative encoding task than in the other encoding tasks across all age categories because these traits are processed at a shallow level (Cited in Rudolph, 1993). The young-referent encoding task and old-referent encoding will be recalled at a more equal processing level between the self-referent and evaluative tasks. We predict the undergraduate students (18-25 years) will view the aged more negatively, meaning they will recall a greater percentage of negative traits than 
positive traits which had been paired with the old-referent encoding task in accordance with Perdue and Gurtman's (1990) results. The non-traditional students (26-54 years), whom have not previously been categorized independently in other studies, will also recall a greater percentage of negative traits than positive traits paired with the oldreferent encoding task because they are students on campus and will identify with the undergraduate population rather then the retired group. This would be consistent with the findings of Mosher-Ashley and Ball (1999) who found no significant differences of ageist attitudes between undergraduates and non-traditional ages. We predict the retired senior citizens (55+ years) will also recall a greater percentage of negative traits than positive traits paired with the old-referent encoding task due to the concept of subjectivity of age. Montepare and Lachman (1989) found older adults' subjective age identities (that is, how they feel) were younger than their chronological age and young adults' subjective age identities were older than their chronological age (Cited in Erber, 2005, p.11). Therefore, the retired senior citizens will not identify themselves as "old" and will create the same barrier seen in the younger populations against older adults.

\section{Method \\ Participants}

A total number of 87 participants comprised the three age categories. Thirtysix participants were undergraduate students, who were enrolled in introductory psychology classes, and between the ages of 18-25 with 29 females and 7 males. These participants received extra credit as compensation for their participation. Twenty-one participants were between the ages of 26-54 with 15 females and 6 males, comprised of non-traditional students. These participants were induced with the use of a lottery drawing of a gift card to the student union. Thirty participants were at least 55 or older with 21 females and 9 males comprised the retired senior citizen age category. All of the participants were Caucasian. Individuals were affiliated with a university of approximately 11,000 students in a Midwestern city of approximately 60,000 people. No restrictions on participants applied; all participant scores were measured and recorded. Participants were treated in accordance with the "Ethical Principals of Psychologists and Codes of Conduct" (American Psychological Association, 1992).

\section{Apparatus}

Participants viewed the encoding task on a projected screen from a Compaq laptop computer; model number Evo N800v, which was programmed using Super Lab Pro. The projector, model number NEC VT670, was property of the university psychology department.

\section{Procedure}

The following research was an extension of the study done by Perdue and Gurtman (1990). Twenty negative and 20 positive trait adjectives were selected from Anderson's (1968) evaluative rankings of 555 trait adjectives. These traits were chosen because of their unambiguous nature, either clearly positive or clearly negative in connotation (Cited in Perdue \& Gurtman, 1990, p. 203). For example, honest was used as a positive trait and cruel was used as a negative trait.

Participants answered one of four possible questions about the adjectives; these are identically replicated from the template study performed by Purdue and 
Gurtman (1990): “(1) Is this a term that would describe you (Self-referent encoding task)? (2) Is this a good trait to find in a person (Evaluative encoding task)? (3) Is this a term that would describe an old person (Old-referent encoding task)? (4) Is this a term that would describe a young person (Young-referent encoding task p.203)?" The self-referent and evaluative questions were included to serve as control conditions so the study did not specifically appear to be studying ageism. The two questions also served to make the needed comparisons between age categories (p. 203).

Encoding questions and traits were randomly paired and presented by computer and viewed on an overhead projector screen. The paired trait and encoding question were presented in 24 point, Arial (Western) font, in black ink against a white background for 6 seconds with a plain white screen appearing between each slide for 2 seconds. Participants bubbled in a "yes" or "no" response to each question on a data sheet using a pen or pencil. Each group judged a random selection of five positive and five negative traits in each of the four encoding tasks for a total of 40 questions.

Testing occurred in a student union at a university and at a retirement home in a closed room varying the time of day and week. Groups no larger than 10 participants were tested at a time. Four versions of the slide show encoding task were made. Counterbalancing was used by systematically varying the pairing of five words with each of the four possible questions used and the order of the questions.

In an unexpected recall task at the end of the session, participants were asked to write down as many traits from the preceding slide show as they could remember. "The probability of recall was defined for each trait as the number of times it was recalled in each condition divided by the number of times it was presented in that condition (Purdue \& Gurtman, 1990, p.203). In this study the probability of recall was computed in percentages.

\section{Results}

The mean total of recalled words was analyzed with a one-way analysis of variance. As shown in Figure 1, the mean total of recalled words from the retired senior citizen age category was significantly lower than the undergraduate and non-traditional age categories, $F(2,84)=14.18, p<.05$. An alpha level of .05 was used for all statistical tests. Because of this significant difference, all further statistical means were reported in mean percent of words recalled.

The mean percent of words recalled was computed for the positive and negative words in each of the four encoding tasks in the three age categories. The means were analyzed with a 3 x 2 x 4 (Age Category X Trait Connotation X Encoding Task) mixed analysis of variance with repeated measures over trait and task. Overall, as shown in Figure 2, no significant difference in mean percent words recalled was found between the three age categories regarding encoding task, $F(6,252)=.58, p>.05$. There was no significant difference in mean percent words, both negative and positive, recalled in any of the four encoding tasks in each of the three age categories, $F(6,252)=.58, p>.05$ (see Figure 3 ).

However, as shown in Figure 4, overall, mean percent positive words recalled was significantly higher than negative words recalled regardless of age category or encoding task $F(1,84)=7.53, p<.05$.

As shown in Table 1, the analysis of variance indicated an interaction between encoding task and trait connotation, $F(3,252)=4.27, p<.05$. Planned 
comparisons indicated that the mean percent of positive words recalled in the self-referent encoding task was significantly higher than those of the negative self referent, $(p<.001)$. No significant differences emerged between negative and positive words for the old $(p=.723)$, young $(p=.369)$, nor evaluative $(p=.176)$ encoding tasks, (see Figure 5). There were no other significant differences among encoding tasks.

Table 1

Analysis of Variance Summary Table Mean \% Words Recalled

\begin{tabular}{llllll} 
Source & SS & DF & MS & F & P \\
\hline Age Category & 1.47 & 2 & .74 & 1.19 & $>05$ \\
Error & 52.08 & 84 & 62 & & \\
Encoding Task & 791.01 & 3 & 263.67 & 1.83 & $>05$ \\
Error & 40706.70 & 282 & 144.35 & & $<.01$ \\
Trait Connotation & 918.51 & 1 & 918.51 & 7.53 & \\
Error & 10251.36 & 84 & 122.04 & & \\
Encoding x Trait & 1164.24 & 3 & 388.08 & 4.27 & \\
Error & 22916.88 & 252 & 90.94 & & \\
\hline Total & & & & & \\
\hline
\end{tabular}

Nate. Significance was found in the trait connotation factor and in the interaction between

trait connotation and encoding task. Both were significant at $p<.01$ alpha level.

\section{Discussion}

Despite previous research findings of higher recall in self-referent and lower recall in evaluative encoding tasks, this study found no significant difference between these two tasks in the separate age categories. Similarly, there was no significant difference between words, positive or negative, recalled from the old encoding task. Since the research on ageism reports conflicting results, this study supports the body of research findings showing that ageism does not exist in the current population. However, the results of this study do not support the original findings of Perdue and Gurtman (1990) or the hypothesis that all three age categories would recall more negative than positive words in the old encoding task revealing ageist attitudes.

When combining age categories and looking at the overall words recalled, it was found that positive words recalled in the self-referent encoding task were significantly higher. This is consistent with the research findings on recall of self-referent words. Memory for self-referent information has been found to be better because it is processed at a deeper level. However, this was not found for negative words in the selfreferent encoding task in this study contrary to the previous research findings. There was no significant difference between positive and negative words recalled in the old, young, and evaluative encoding tasks. 
Counterbalancing the stimuli was effective by randomly varying the 20 positive and 20 negative words with each of the four encoding tasks. Varying the time of day and day of week successfully controlled for the possible confound of subject selection. By using a pre-tested list of trait adjectives, all words were clearly positive or clearly negative in connotation. Each word and encoding question was shown for the same amount of time on a projected computer screen.

Participants were not evenly distributed by age category among the four encoding task programs because of the availablility of participants and the restricted testing schedule. By lengthening the duration of testing to allow for more testing times, a better distribution of participants could be achieved. It may also be more beneficial to test participants individually, which would allow for more control over the version to which each participant was exposed.

The uniqueness of our study was that it tested ageism across adulthood. However, when measuring the dependent variable of words recalled, there may have been a scale-attenuation effect in the retired senior citizens age category. Since recall for this group was significantly lower than the other age categories, the study may have been confounded by a floor effect. Perdue and Gurtman did not encounter this confound in their study because their study only tested the undergraduate population. Knowing that free recall is a difficult task, it may be warranted to change the dependent variable to words recognized. It may also be warranted to consider other methodologies for testing implicit ageism across adulthood to account for irreversible developmental age-related slowing and memory deficits.

The majority of the results are specific to the population of college-educated Caucasians. The results from this experimental population do not generalize to all populations and cannot be representative of holistic societal views. To further generalize the results, the pooling of participants should not be restricted to a university campus; diverse ethnic and educational backgrounds should be included in the sample studied. By expanding the participant pool into a larger community, the external validity of this study would be increased.

There may be gender differences in ageist attitudes. Weinberger and Millham (1975) found females held more negative attitudes toward the aged than did males in the undergraduate student age group. This is consistent with the findings of Kalavar (2001), in her study analyzing the difference in attitudes between male and female college students using an age preference task. Due to the low participation of males in this study, gender differences were not considered a factor; however, Kalavar (2001) states, "Gender socializtion may play an important role in the formation of ageist attitudes. Further research in this area of gender differences is strongly recommended (p. 512)."

Past studies have failed to test ageism across adulthood. This study has contributed to the science of psychology by expanding the sample population tested to include non-traditional aged students and retired senior citizens in addition to the traditionally tested undergraduate population. Research on ageism is important because discriminatory thoughts and behaviors against older adults are wrong and detrimental to society's growth. Converging evidence is needed to resolve the current conflicting findings on whether or not ageist attitudes are present in today's society. This research has increasing importance, overall, as the age demographics change and the baby boomer cohort moves into older adulthood. 


\section{Appendix}

Figure 1. Total number of words recalled as a function of age category. The mean total of recalled words from the retired senior citizen age category was significantly lower than the undergraduate and non-traditional age categories, $F(2,84)=14.18, p<.05$; lines on bar graph indicate $\pm 1 S E$.

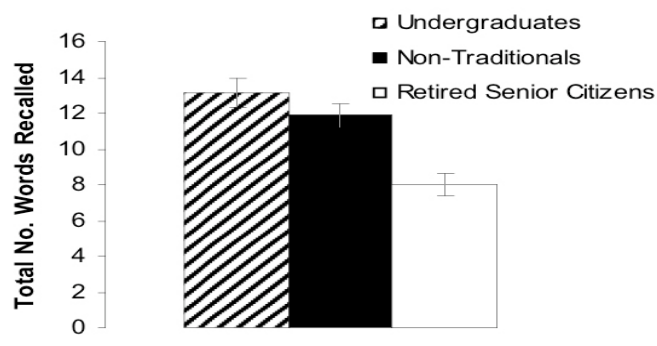

Age Category

Figure 2. Mean percent positive and negative words recalled as a function of encoding task in the three age categories. Undergraduates (Top). Non-traditionals (Middle). Retired Senior Citizens (Bottom). No significant difference in mean percent words recalled was found between the three age categories regardless of encoding task, $F(6,252)=.58, p>.05$; lines on bar graph indicate $\pm 1 S E$.
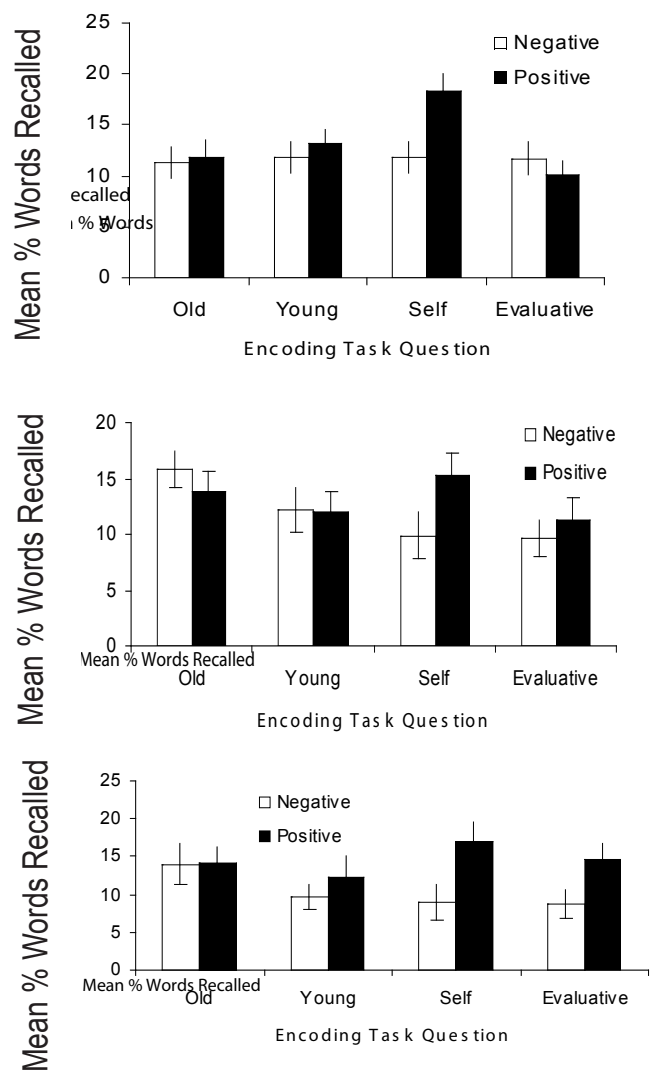
Figure 3. Mean percent words recalled in old, young, self, and evaluative encoding tasks as a function of age category. (Top) Undergraduate. (Middle) Non-traditional. (Bottom) Retired Senior Citizens. There was no significant difference in mean percent words, both negative and positive, recalled in any of the four encoding tasks across all age categories, $F(6,252)=.58, p>.05$ (see Figure 3); lines on bar graph indicate $\pm 1 S E$.
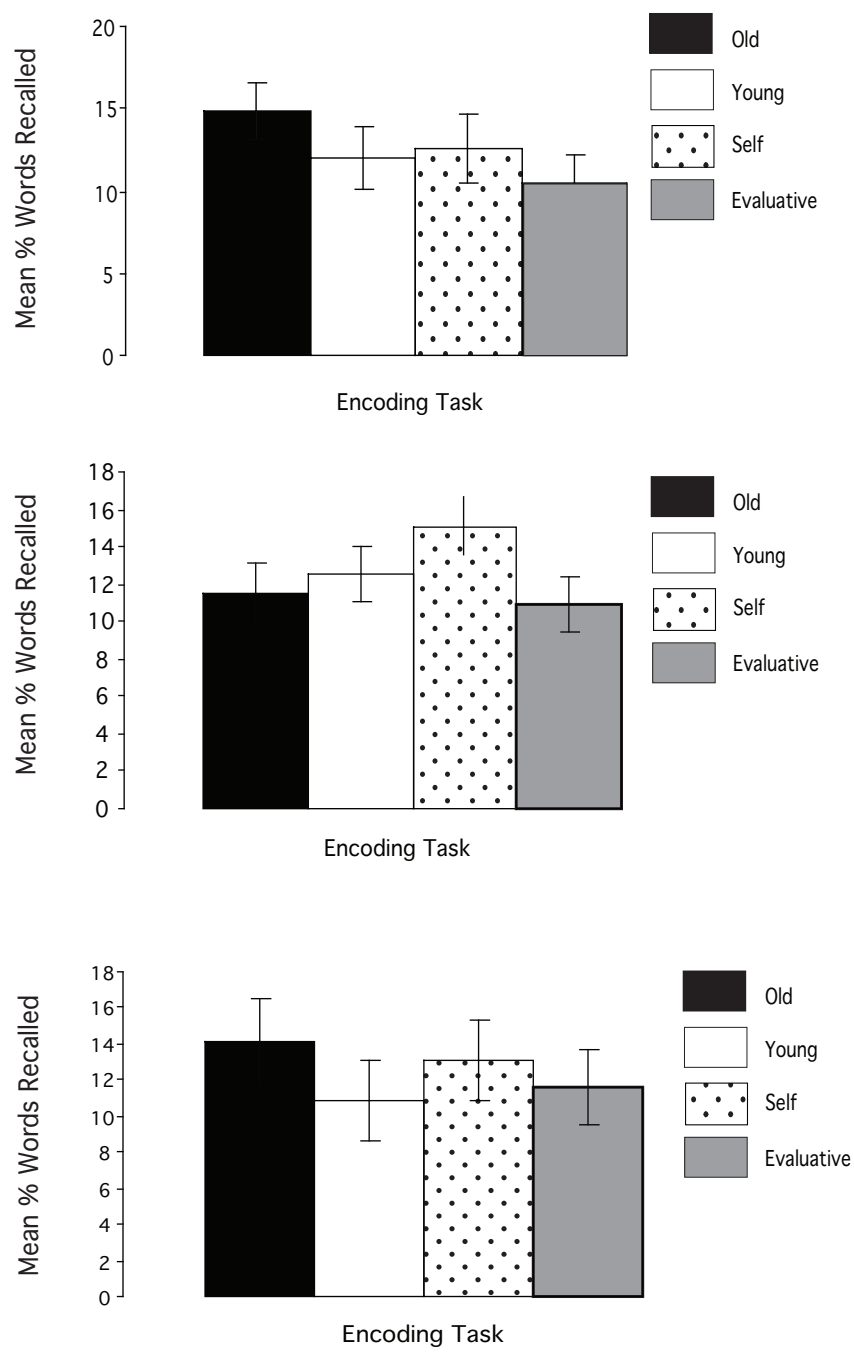
Figure 4. Mean percent positive and negative words recalled as a function of encoding task. Mean percent of positive words recalled in the self referent encoding task was significantly higher than those of the negative self referent, $(p<.001)$ There were no significant differences between negative and positive words for old ( $p=.723)$, young ( $p=.369)$, and evaluative ( $p=.176)$ encoding tasks. (see Figure 5). There were no other significant differences among encoding tasks. Lines on bar graph indicate $\pm 1 S E$.

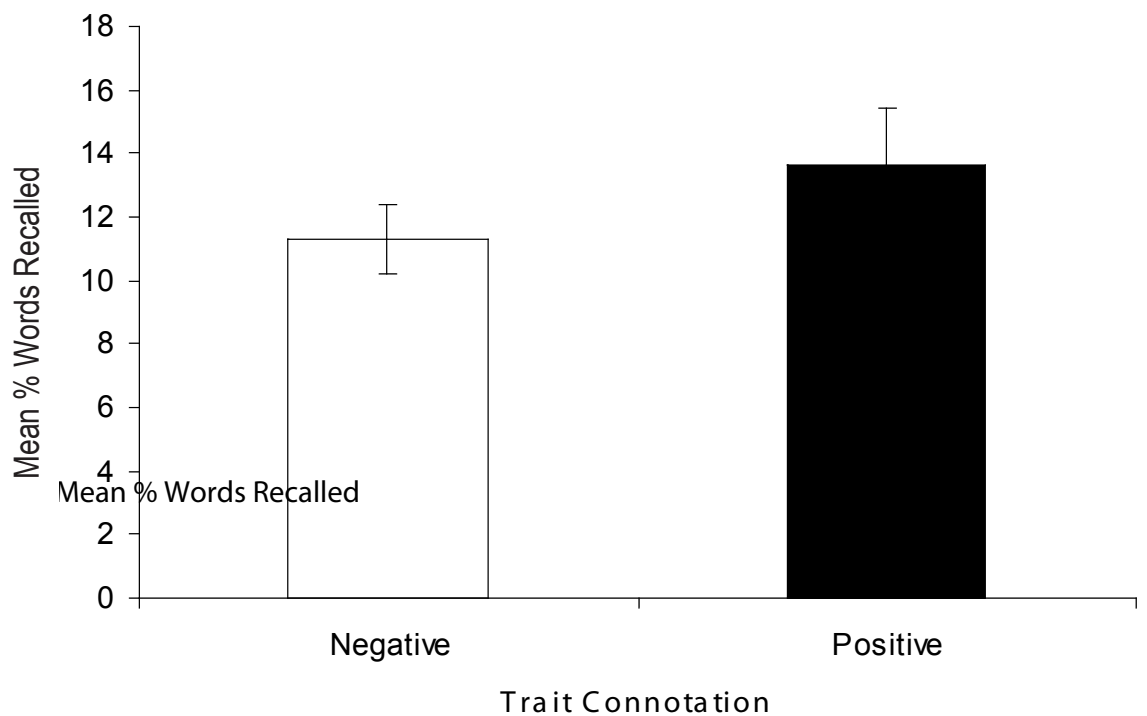

Figure 5. Mean percent words recalled as a function of trait connotation. Mean percent positive words recalled was significantly higher than negative words recalled $F(1,84)=7.53, p<.05$; lines on bar graph indicate $\pm 1 S E$.

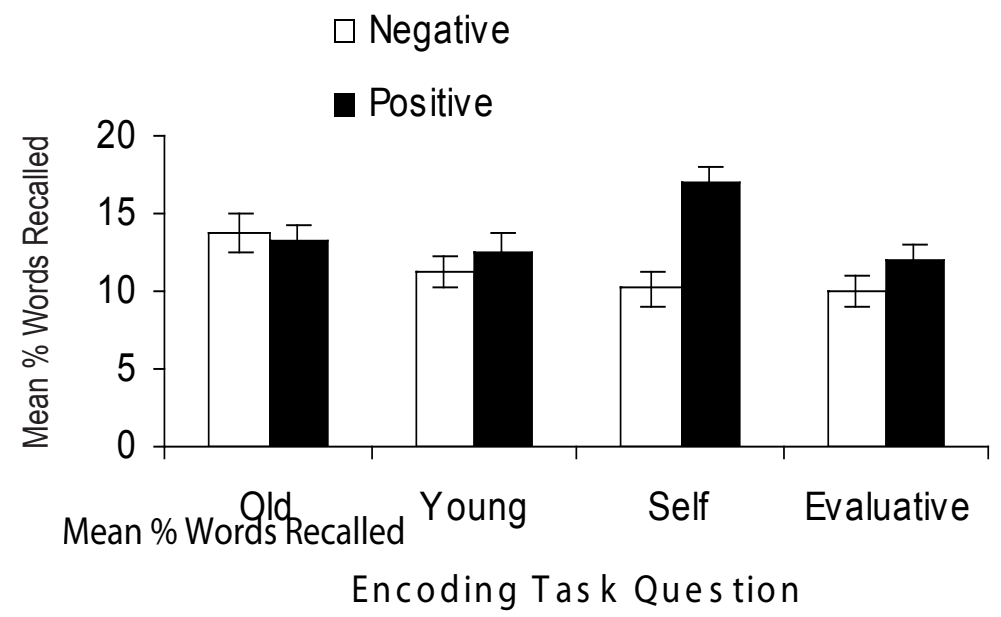




\section{References}

Aaronson, B.S. (1966). Personality stereotypes of aging. Journal of Gerontology, 21, 458-462.

American Psychological Association. (1992). Ethical principals of psychologists and code of conduct. American Psychologist, 47(1), 1597-1611.

Anderson, N.H. (1968). Likeableness ratings of 555 personality-trait words. Journal of Personality of Social Psychology, 9, 272-279.

Bell, B.D., \& Stanfield, G.G. (1973). Chronological age in relation to attitudinal judgments: An experimental analysis. Journal of Gerontology, 28(4), 491-496.

Bonnesen, J.L., \& Burgess, E.O. (2004). Senior moments: The acceptability of an ageist phrase. Journal of Aging Studies, 18(2), 123-142. Retrieved February 18, 2005, from PsychARTICLES database.

Butler, R.N. (1978). Age-ism: Another form of bigotry. In M.M. Seltzer, S.L. Corbelt \& R.C. Atchley (Eds.), Social Problems of the aging. Blemont, CA: Wadsworth.

Carver, C.S., \& de la Garza, N, H. (1984). Schema guided information search in stereotyping of the elderly. Journal in Applied Social Psychology, 14(1), 69-81. Retrieved February 18, 2005, from PsychARTICLES database.

Erber, J.T. (2005). Aging \& older adulthood. Belmont, CA: Wadsworth.

Hawkins, M.J. (1996). College students' attitudes toward elderly persons. Educational Gerontology, 22(3), 271-279. Retrieved February 18, 2005, from PsychARTICLES database.

Hoyer, W.J., \& Roodin, R.A. (2003). Adult development and aging: fifth ed. New York: McGraw-Hill.

Hummert, M. L. (1990). Multiple stereotypes of elderly and young adults: A comparison of structure and evaluations. Psychology and Aging, 5(2), 82-193. Retrieved February 18, 2005, from PsychARTICLES database.

Kalavar, J. M. (2001). Examining ageism: Do male and female college students differ? Educational Gerontology, 27, 507-513. Retrieved March 20, 2005, from PsychARTICLES database.

Middlecamp, M., \& Gross, D. (2002). Intergenerational daycare and preschoolers' attitudes about aging. Educational Gerontology, 28, 271-288. Retrieved February 18, 2005, from PsychARTICLES database.

Mosher-Ashley, P.M., \& Ball, P. (1999). Attitudes of college students toward elderly persons and their own perceptions of themselves at age 75. Educational Gerontology, 25(1). Retrieved February 18, 2005, from PsychARTICLES database.

Payne, D.G., \& Wenger, M.J. (1998). Cognitive psychology. Boston, MA: Houghton Mifflin Company.

Polizzi, K.G. (2003). Assessing attitudes toward the elderly: Polizzi's refined version of the aging semantic differential. Educational Gerontology, 29, 197-216. Retrieved February 18, 2005, from PsychARTICLES database.

Purdue, C.W., \& Gurtman, M.B. (1990). Evidence for the automaticity of ageism. Journal of Experimental Social Psychology, 26, 199-126. 
Rudolph, U. (1993). The self-reference effect: Methodological issues and implications from a schema-theoretical perspective. European Journal of Social Psychology, 23, 331-35. Retrieved February 18, 2005, from PsychARTICLES database.

Sheomake, A.F., \& Rowland, V.T. (1993). Do laboratory experiences change college students' attitudes toward the elderly? Educational Gerontology, 19, 295-309.

Slotterback, C.S. (1996). Projections of aging: Impact of generational differences and the aging process on perceptions of adults. Psychology and Aging, 11(3), 552-559. Retrieved February 18, 2005, from PsychARTICLES database.

Sorgman, Margo I., \& Sorensen, Marilou. (1984). Ageism: A course of study. Theory Into Practice, 23(2), 117-124. Retrieved February 18, 2005, from PsychARTICLES database.

Weinberger, L.E., \& Millham, J. (1975). A multi-dimensional, multiple method analysis of attitudes toward the elderly. Journal of Gerontology, 30(3), 343-348 